\title{
Imaging Informatics: from Image Management to Image Navigation
}

\author{
0. Ratib \\ Department of Medical Imaging and Information Sciences, University Hospital of Geneva, Switzerland
}

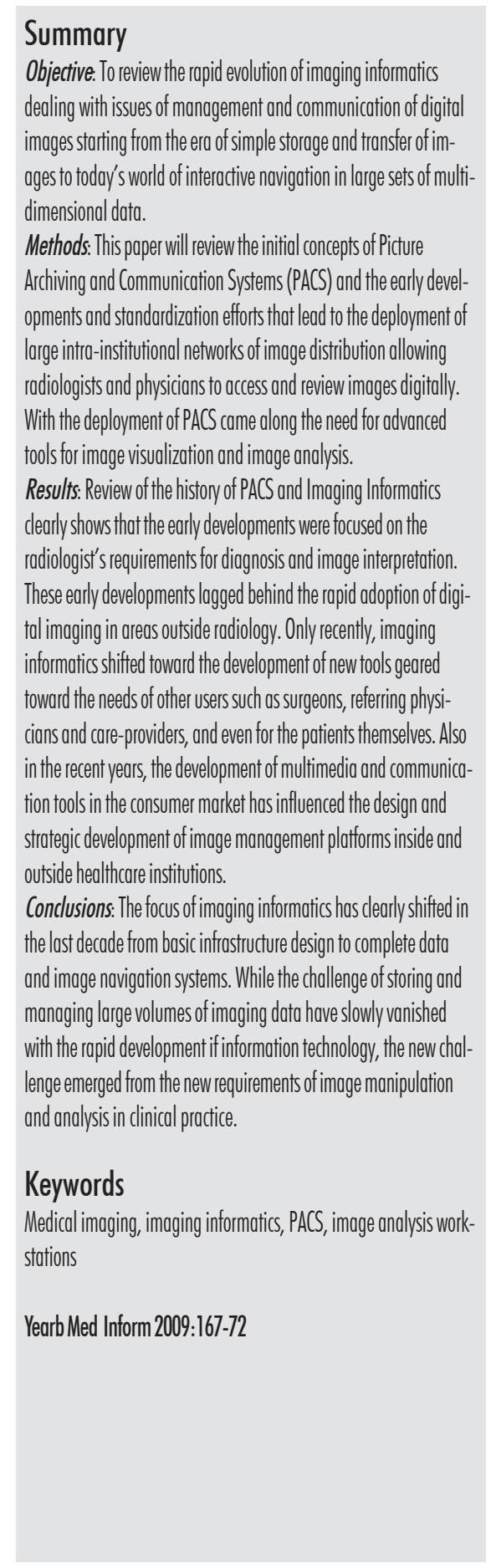

\section{Introduction}

In the early eighties, emergence of digital imaging in medicine brought a new challenge to computer scientists to find ways of managing increasingly large volumes of image data in digital format. The conversion of conventional Xrays from traditional films to digital detectors generated very large size images that required adequate display technology that was beyond the capabilities of standards CRT at that time. Conversely, a rapid evolution of scanners technology raised the number of images and the resolution that can be acquired resulting in a huge growth in data volumes. This rapidly growing field led to tremendous interest among the scientific and research community. The earliest reference to PACS that we know of came in 1979 when professor Heinz Lemke, PhD, at the Technical University of Berlin published a paper on applied image processing and computer graphics methods in a study of head CT scans [1]. In his paper he described all the basic components including interfaces to hospital information systems. Soon after, a first pivotal event was a SPIE/PACS conference held in 1982 in Los Angeles under the initiative of Dr. André Duerinckx and Samuel J. Dwyer III, PhD, internationally recognized pioneers and leaders in this emerging field [2]. It was at this conference that the acronym PACS for Picture Archiving and Communication Systems was first cited and used ever since. A recent article published in 2003 [3] reports a transcript of the original workshop of 1982. In the following years this new discipline has slowly matured through different early projects in the US and in Europe aimed toward the development of systems capable of handling large volumes of imaging data and pro- vide the necessary tools for radiologists to review them and make adequate diagnosis. These early home-grown systems were clumsy and expensive, but they lead the way to the concept of a filmless radiology.

\section{The Pioneers}

Early adopters of this new concept developed several innovative and unprecedented systems that were tested in the field and in real clinical practice. Samuel J. Dwyer 11l, an electrical engineer working on digital imaging, who was one of the co-organizer of the landmark PACS conference in Los Angeles, oversaw the building of what is probably one of the first PACSat the University of Kansas. Based on connecting ultrasound machines and film digitizer in an Ethernet network connected to a handful of workstations served as a demonstration system and a proof of concept. At the same time, Dr. Steven Horii who also attended the 1982 PACS conferenceand who was then at New York University, had been working on a project to create side-by-side digital displays of nuclear medicine head scans and head CTs. Due to the difficulties he encountered in reading encrypted proprietary image formats of the CT studies he soon realized that a more open standard for images would be necessary. He later became instrumental in standardization efforts and one of the promoters of the DICOM initiative (see below). At UCLA another pioneering effort was under way that came to fruition between 1989 and 1991 under the leadership of H.K. "Bernie" Huang, DSc, FRCR, that had come to UCLA in the early 1980 s and started a new medical imaging division and a graduate program in medical physics [4]. Prof. Huang put his graduate students, who included myself, to 
work on building a homegrown PACS. It was initially deployed in pediatric radiology and used early low-resolution monochrome monitors. It was built around the first two CR units in the United States allowing direct capture of digital Xray images. More significantly, from a manufacturing source he obtained two computer boards that enabled him to decode the digital information on the CR plates. This made it possible to display the x-rays on the PACS monitors. Later, UCLA became one of the first sites to show the use of high-resolution monitors for PACS workstations capable of displaying images at full resolution of 2000x2500 pixels and a dynamic depth of 10 bits. At the University of Florida, Gainesville, Janice Honeyman-Buck was also struggling in the early 1990s to complete a limited PACS. With her group she attempted to break the code and extract CT and MR image data from the scanners way before DICOM standard came along, to store these images in a central archive built on common jukebox of large optical disks.

At the same time a small group of early adopters in Europe have initiated several exploratory projects [5]. Professor A.R. Bakker at the university of Leiden was one of them. Together with Professor Heinz Lemke from Berlin and a handful of others they created the first PACS society "EuroPACS" in 1984. Bakker and his co-workers at BAZIS in Leiden carried out PACS research and development as part of hospital information systems (HIS) activities. BAZIS, a non-profit organization, developed and supported an integrated hospital information system widely in use in hospitals in the Netherlands. In 1986, Dr. M. Osteaux and his team at the University Hospital of Brussels together with the Pluridisciplinary Research Institute for Medical Imaging Science (PRIMIS) started one of the few multi-vendor PACS installations worldwide [6]. At the same time at the University Hospital in Geneva a new PACS architecture with distributed archive and interactive image visualization workstation [7] was developed as an extension to the existing hospital information system (HIS). Under the leadership of Prof.
J.R. Scherrer the creator of the Diogene hospital information system [8], Dr. O. Ratib and his team developed their own image management system based on a distributed architecture [9] with an innovative imaging workstation called Osiris based on interactive graphic user interface (GUI) that was developed to be portable on different operating systems such as Unix, Microsoft Windows and Apple computers [10]. Other projects were also attempted in other countries such as Austria, France, Italy and United Kingdom [11]. In the latter, some ambitious projects were envisioned as early as 1982 were plans to create the first filmless hospital in the United Kingdom at St Mary's Hospital in London were submitted by Dr J.O.M.C. Craig of St Mary's with the Regional Scientific Officer, Harold Glass.In same time in France a very early PACS project (called SIRENE) was initiated by Dr J.M. Scarabin, a neurosurgeon in Renne's University Hospital and Dr R. Renoulin, an engineer working in the field of enterprise networks in a Telecommunications Research Center in Rennes. The EuroPACS association (http://www. europacs.org/) supported by all the early leaders of these projects has continued to pursue its original mission of promotion and education of new concepts in PACS and medical imaging and continues today to be one of the leading international organization in the field holding annual meetings and educational seminars and workshops.

The early projects suffered from significant limitations in performance and were only able to handle a limited number of simultaneous users and relatively small volumes of data. Complex image management mechanisms and routing algorithms were created for pre-fetching and pre-loading image files on selected workstations [12]. Users were bound to follow predefined distribution rules and workflow. With such complex communication models these systems had limited scalability and could not easily adapt to different institutions and were soon abandoned.

These early prototypes developed in centers in Europe and in the United States were soon challenged by emerging commercial systems. In many early implementations joint developments and collaborative efforts were initiated be- tween academic centers and industry. With this trend came the urge for standardization efforts allowing multi-vendor systems to interconnect.

\section{Emergence of Standards}

The need for a standard allowing interconnectivity and exchange of images between devices from different manufacturers led the American College of Radiology (ACR) and the National Electrical Manufacturers Association (NEMA) to form a joint committee in order to create a standard method for the transmission of medical images and their associated information. This committee, formed in 1983, published in 1985 the ACR-NEMA Standards Publication No. 300-1985s. While the initial versions of the ACR-NEMA effort (version 2.0 was published in 1988) created standardized terminology, an information structure, and unsanctioned file encoding, most of the promise of a standard method of communicating digital image information was not realized until the release of version 3.0 of the Standard in 1993. The release of version 3.0 saw a name change, to Digital Imaging and Communications in Medicine (DICOM), and numerous enhancements that delivered on the promise of standardized communications [13]. The DICOM Standard now specified a network protocol utilizing TCP/ IP, defined the operation of Service Classes beyond the simple transfer of data, and created a mechanism for uniquely identifying Information $\mathrm{Ob}$ jects as they are acted upon across the network. DICOM was also structured as a multi-part document in order to facilitate extension of the Standard. Additionally, DICOM defined Information Objects not only for images but also for patients, studies, reports, and other data groupings. With the enhancements made in DICOM (Version 3.0), the Standard was now ready to deliver on its promise not only of permitting the transfer of medical images in a multivendor environment, but also facilitating the development and expansion of picture archiving and communication 
systems (PACS) and interfacing with medical information systems.

In the mid nineties, all vendors started to adopt DICOM standard in their products facilitating the development of PACS and the exchange of images in digital form between modalities and interpretation workstation. "Soft copy reading" on computer screens slowly replaced traditional film reading in most radiology departments. With the development of PACS came the need for better workflow management and efficient delivery of information and images where needed by the radiologists and physicians. Different scenarios were applied in different settings resulting in very heterogeneous and often inefficient and complex systems. In order to streamline their products and unify their strategies, vendors needed more specific guidelines and detailed specifications on clinical workflows. This lead to the IHE initiative for standardization and documentation of image management workflow in clinical settings [14]. Integrating the Healthcare Enterprise (IHE) is an initiative by care providers (including ACC, HIMSS and RSNA) and vendors to improve the way information systems communicate to support patient care. IHE defines Integration Profiles that use established data standards to integrate systems for effective interoperability and efficient workflow. IHE makes it possible to achieve the level of integration required in the era of the electronic health record. Each IHE Integration Profile describes a clinical requirement for systems integration and a solution to address it. It defines functional components, called IHE Actors, by specifying in careful detail the transactions, based on standards such as DICOM and HL7, each Actor must perform. IHE provides specification for a more unified framework for implementation of integrated PACS with HIS and RIS.

\section{From Prototypes to Professional Systems}

As commercial PACS systems started to appear on the market they were soon adopted by some early adopter in large institutions in the United States, in Europe and in Asia. In 1993 Dr. Eliot Siegel, now vice chairman and professor of diagnostic radiology at the University of Maryland was the first to take on the challenge of building a totally filmless hospital. At the same time Steve Horii and his team who were heavily involved in the development of the DICOM standard also initiated a collaborative effort with industry to implement a commercial system at the University of Penn. Professor H.K. Huang who joined Professor Ronald L. Arenson, chairman of the radiology department in the University of California at San Francisco medical school, also helped building a clinical PACS while continuing innovative research and developments in imaging informatics [15]. Soon after, in 1998, under the leadership of Prof. O. Ratib who was appointed as vice chair of the department of radiology at the University of Los Angeles Medical Center, UCLA migrated from two generations of home-built PACS to a commercial system with one of the first off-site long term archive based on an Archive Service Provider(ASP) model [7]. An innovative approach where the responsibility of long term storage of images was outsourced to a vendor providing large capacity storage service based in data centers located remotely from the medical centers. A similar approach was adopted by Northwestern Medical center in Chicago under the leadership of Dr. David Channin who was responsible of enterprise-wide image management and PACS with a strong commitment to IHE guidelines. In Europe, in Graz Austria, following pioneer work of Professor G. Gell on PACS and RIS integration, and ambitious project for a fully filmless hospital based on a commercial PACS was launched in the new Danube Hospital (formerly SMZO) in Vienna under the leadership of Professor Hruby chairman of the department of radiology. In Italy after some pioneer work strongly geared toward teleradiology at the university of Trieste, and university of l'Aquilla, a collaborative effort with industry for implementation of PACS and teleradiology was undertaken at the uni- versity of Pisa under the leadership of Professor. D. Caramella. In Asia, following early pioneer work starting in 1984 in Osaka university and in 1988 in Hokkaido university, the first large scale PACS projects started in the early nineties. In 1994, at Osaka University, Pr. K. Inamura and his team installed one of the first clinically operational PACS implemented in an environment where HIS and RIS were already operational. After a rapid growth in number of PACS in the following years a recent review of the history of these installations show that a number of projects were abandoned due to poor performance and inadequate quality [16]. With the advent of new commercial systems and higher network bandwidth and better workstations a rapid growth in the number of institutions implementing PACS between 1997 and 2002 show an exponential growth curve. In Korea a rapid deployment of PACS in public and private hospital was driven by a government inventive policy favoring institution that would implement PACS systems and favor private industry that would invest in PACS, resulting in several local small industry to invest in numerous large full-scale PACS projects. In the recent years a large nationwide PACS and teleradiology network project was launched in Hong Kong by the Hon-Kong Hospital authority IT department in collaboration with Prof. H.K. Huang team. The Hong Kong Wide Area Image Distribution/PACS Project covers 44 public hospitals and 51 specialized outpatient centers.

\section{Academics Pushing the Limits}

While industry slowly penetrated the market with commercial PACS solution that responded to the initial needs for image management and image interpretation on digital workstations it lagged behind the rapid increase in demand and changes in clinical requirements. This gap between commercial systems and the clinical demand was due to the lack of clear guidelines and specifications from the medical community due to unanticipated changes in paradigm and 
accelerated growth in utilization and development of imaging techniques in clinical practice. This growth and rapid change in requirements also coincide with a drastic change in most industrialized countries were healthcare policies were heavily driven by economic constrains and explosion of costs driving governments and agencies to impose new management strategies driving toward higher productivity at lower costs.

Research for innovative and more efficient solution was mostly carried in academic institutions where close interactions between clinical settings and technical research was possible. With the rapid shift in workflows and changes in clinical practice new requirements triggered innovative and sometime disruptive solutions to emerge. Major efforts were invested in exploring new techniques that will allow faster transfer and wider distribution of images in institutions where image data were needed not only within radiology departments but also in clinical wards, specialized services, surgical suites etc. Also, because images were needed more and more outside radiology a change in requirements of workstation features was also driven beyond the traditional PACS workstation intended for image interpretation and primary diagnosis by radiologists. Early work by Dr. Paul Chang at the University of Pittsburg and later distributed commercially by Stentor Inc., brought a completely new and innovative approach to image management [17]. Focusing on the performance and speed of image access anywhere-any-time Dr. Chang and his team explored new ways of compressing and transmitting large image sets from storage devices to workstations [18]. Progressive compression and decompression of images associated with advanced data streaming techniques, provided faster and almost real-time access to images while allowing full resolution rendering to be maintained dynamically. This pioneer work has changed the rules and initiated new trends in image management and communication replacing traditional pre-fetching and point to point transmission of images to a more distributed access to images when they are need where they are needed. This change in paradigm was also supported by the rapid evolution of web-based technologies allowing faster data streaming of very large data sets as well as facilitated access to high performance data processing through thin-client web-based front ends.

Another area where academic research has driven new changes in design of image management and PACS solutions is in the area of image analysis and image processing. Innovative techniques are slowly appearing in the field of computer-assisted diagnosis providing quantitative and automated techniques that help radiologists in interpretation and analysis of images improving their accuracy and performance. But the area of image interpretation is not he only area where the rapid development of new tools was making rapid progress. In the field of $3 \mathrm{D}$ rendering and image visualization, new paradigm of image display and image processing started to emerge with solution tailored more for physicians outside radiology departments such as surgeons, oncologists, cardiologists, and referring physicians that need image data for patient management and clinical decisions. The increasing number of interventional procedures relying on image guidance and the unprecedented evolution of minimal invasive surgery techniques require high resolution images to be available in many clinical settings outside radiology. Image access in operating rooms and interventional suites set a new level of requirements for image management and communication [19]. Professor Heinz Lemke has advocated, for many years now, the need for special architecture and workflow modeling in operating rooms, which lead him to suggest a new usage of the PACS acronym for "Picture Archiving and Communication in Surgery" [20]. The specific requirements for image management in operating suites, lead to the launch of a new extension of the DICOM standard for surgery. These new standardization efforts are carried out by the working group 24 of the DICOM committee under the leadership of Prof. Lemke.

In an effort to better handle image access in large and complex system, some advanced and innovative context-based image retrieval (CBIR) based on ontology were developed $[21,22]$ to enrich the core functionalities of picture archiving and communication systems [23]. The goal of such systems being to access and retrieve images by content rather than by alphanumeric-based indices. Although this approach was originally developed for multimedia repositories such as those on the World Wide Web, techniques for content-based access to medical image repositories are subject of high interest in research as well as clinical applications. More specific applications of ontology and semantic models have also been adopted to better describe the structure and content of diagnostic imaging studies. Ontological models were used to represent radiological and clinical knowledge to integrate PACS other clinical information systems and to support radiology interpretation process [24].

More specific developments for linking broader research activities and hosting collaborative efforts in medical imaging have also emerged from academic and scientific communities around the world. An example of such coordinated effort is the Biomedical Informatics Research Network (BIRN, www.nbirn.net) which mission is to construct the infrastructure for gathering, organizing and managing knowledge, and developing a federated architecture for linking multiple databases across sites contributing data and knowledge [25]. At the core of this knowledge organization is BIRNLex, a formally-represented ontology facilitating data exchange tool providing a means to cast queries against specific data sources in the federation

Medical imaging is has also evolved toward functional and molecular imaging modalities that are able to produce images that represent an in-vivo characterization of biological processes. Positron Emission Tomography (PET) using radio-labeled tracers represents the most common molecular imaging modality, but other techniques based on molecular markers, are nowadays emerging in other modalities such as MRI offering new perspectives of functional imaging. The ability to combine the func- 
tional or molecular data with the anatomical images adds a new dimension to the images. Adding new dimensions to the images such as time varying parameters (often referred to as the fourth dimension) and functional and metabolic parameters (referred to as the fifth dimension) require special navigation tools and multidimensional rendering software that are usually not available on traditional PACS workstations. Such visualization tools are often only available on high-end 3D rendering workstations in academic settings and specialized radiology departments. Ironically, where these tools are needed the most is in clinical services and in areas where clinical decisions and patient management choices are being made. They are also becoming important communication means for radiologists to transmit their findings to referring physicians. The availability of such tools are however very limited outside radiology departments due to their high cost and complexity.

\section{Open Source: the New Paradigm in Imaging Informatics}

A new paradigm in computer software came with the success of Open Source projects such as Linux, Firefox, OpenOffice and other widely adopted software platforms. The rationale behind open source is very simple: When programmers can read, redistribute, and modify the source code for a piece of software, the software evolves. People improve it, people adapt it, people fix bugs. And this can happen at a speed that, if one is used to the slow pace of conventional software development, seems astonishing. The rapidly growing open source community has realized that this rapid evolutionary process produces better software than the traditional closed model, in which only a very few programmers can see the source code and everybody else must blindly use an opaque set of software tools. This trend also made some progress in medical informatics and some early projects were developed under Open Source licensing, providing source code to the community to share. Some ambitious Open Source project included the Vista project (ref) a home-grown electronic medical record implemented in Veterans Affairs (VA) Hospitals in the United States. The impact of open source is even greater in specialized areas of medicine such medical imaging. These vertical markets have always been a challenge for vendors and manufacturers due to the small size of specialized users and high expectations in terms of complexity and performance of the tools that users need. This has naturally driven the market to high-end and highcost developments and marketing strategies that also try to cope with very rapid evolution of computer technologies and software developments that make most products obsolete in very short time interval, which does not allow the manufacturers to generate sustainable return on investment. Open Source solutions can challenge this limited market share of large industrial vendors and provide more adequate and affordable solutions. A list of recent Open Source initiatives in Medical Imaging Software can be found at (www. idoimaging.com). In the scientific community, a new set of open source libraries have emerged for the visualization of multidimensional data. The most successful set of tools is provided by Insight Software Consortium (www. insightsoftwareconsortium.org): The Visualization Toolkit or VTK (is a well recognized and widely adopted software library that runs on multiple platforms and has been used for numerous scientific and medical applications so far. The recent adjunction of the Insight Toolkit or ITK, mostly funded by the US National Library of Medicine as part of the Visible Human Project, adds a wealth of additional rendering and image processing tools for medical applications. Numerous Open Source software projects providing a variety of medical imaging tools have emerged in the recent years.

One of the most widely adopted Open Source software platform for medical imaging is the Osirix software (www. osirix-viewer.com) that was initiated at the University of California in Los An- geles (UCLA) in 2004 and then carried out in Geneva by two Swiss radiologists Dr. Antoine Rosset and Prof. Osman Ratib, soon joined by Joris Heuberger, a third computer scientist from Geneva [26]. Together they designed and developed this new image-processing platform specifically designed to take advantage of the significant progress in performance and flexibility of 3D rendering and image processing on consumer-market personal computers [27].

The OSIRIX software was tailored to provided physicians unfamiliar with advanced image processing tools with a platform allowing them to manipulate and visualize large sets of image data using advanced volume rendering and three dimensional navigation tools. OSIRIX user interface was designed to allow physicians to rapidly become familiar with the manipulation of 3D objects and navigating through multi-dimensional images. Advanced image processing and analysis tools are being added to the program everyday. Developers from all around the world have contributed to the extension of OSIRIX by adding innovative and specialized image processing features. Furthermore, the OSIRIX software architecture allows for separate processing modules to be added to the program as plug-ins.

\section{Conclusions}

An amazing evolution of imaging informatics occurred in the last couple of decades from the early days where systems for digital image management had to be developed to allow radiologists to review images on soft-copy workstations in replacement of traditional hard-copy films to the recent developments of multimodality image display and analysis software platforms. The early adopters and pioneers in the field have paved the road of today's PACS that are taken for granted by the newer generation of radiologists and physicians. While tremendous progress were made in designing and adopting new and innovative paradigms, the penetration of imaging informatics in medi- 
cal institutions is still lagging behind. Users are still struggling with inappropriate an inefficient tools while they are being challenged by the unmanageable quantities of data generated by modern imaging modalities.

The success of developments in PACS and large institutional projects is in big part due to the efforts deployed by devoted advocates of standards and their tireless work in standardization bodies. Without these standards the development of commercial systems would have never been possible, and users would still be left with homegrown heterogeneous system that could never be integrated with other information systems.

Open source software is an idea whose time has finally come. For twenty years it has been building momentum in the technical cultures that built the Internet and the World Wide Web. Now it's breaking out into the commercial world and into vertical markets such as medical applications, and that's changing all the rules. It is particularly promising in advanced image display and analysis applications where the rapid increase in demand cannot be matched by traditional expensive commercial solutions.

\section{References}

1. Lemke HU, Stiehl HS, Scharnweber H, Jackel D. Applications of picture processing, image analysis and computer graphics techniques to cranial CT scans. In: Computer-aided analyses of radiological images. Newport Beach: IEEE; 1979.

2. Duerinckx AE. First international conference and workshop on picture archiving and communication systems (PACS) for medical application. NewPort Beach: SPIE; 1982.

3. Duerinckx AJ. Introduction and the PACS '82 panel discussions: Panel 1-Equipment manufacturers' view on PACS and Panel 2-The medical community's view on PACS. 1982. J Digit Imaging 2003;16(1):32-68; discussion 29-31.

4. Huang HK, Ratib O, Bakker AB, Witte G. Picture Archiving and Communication System (PACS) in Medicine. NATO ASI Series. Berlin/Heidelberg: Springer-Verlag; 1991

5. Lemke HU. PACS developments in Europe. Comput Med Imaging Graph 2003;27(2-3):111-20.

6. Osteaux M, Van den Broeck R, Verhelle F, de Mey J. Picture archiving and communication system (PACS): a progressive approach with small systems. Eur J Radiol 1996;22(3):166-74.

7. Ratib O, Swiernik M, McCoy JM. From PACS to integrated EMR. Comput Med Imaging Graph 2003;27(2-3):207-15.

8. Geissbuhler A, Lovis C, Spahni S, Appel RD, Ratib $\mathrm{O}$, Boyer $\mathrm{C}$, et al. A humanist's legacy in medical informatics: visions and accomplishments of Professor Jean-Raoul Scherrer. Methods Inf Med 2002; 41(3): 237-42.

9. Ratib O, Lemke H, Trayser G, Vurlod JF, Do H, Scherrer JR. Distributed Image Management and Hierarchical Storage in an Integrated RIS and PACS. In: MEDINFO'92. Geneva (Switzerland): NorthHolland Ed. Amsterdam; 1992

10. Ligier Y, Ratib O, Funk M, Perrier R, Girard C, Logean M. Portable image-manipulation software: what is the extra development cost? J Digit Imaging 1992;5(3):176-84

11. Ratib O, Ligier Y, Scherrer JR. Digital image management and communication in medicine. Comput Med Imaging Graph 1994;18(2):73-84.

12. Bakker AR, Stut WJ Jr, De Valk JP, Reijns GR. PACS costs: modelling and simulation. Med Inform (Lond) 1988;13(4):307-13.

13. Dicom Committee. DICOM strategic document, D.S.C.a.I.W. Groups, Editor; 2008.

14. O’Donnell K. IHE Radiology User's Handbook 2005, ACC/HIMSS/RSNA.

15. Carey B, Seshadri SB, Arenson RL. PACS: "Back to the Future". In: Jost RG, editor: Medical Imaging V:PACS Design and Evaluation. Proc SPIE 1991;1446:414-9.

16. Inamura K, Kousaka S, Yamamoto Y, Sukenobu Y, Okura Y, Matsumura Y, et al. PACS development in Asia. Comput Med Imaging Graph 2003;27(23):121-8

17. Morgan MB, Branstetter BFT, Lionetti DM, Richardson JS, Chang PJ, The radiology digital dashboard: effects on report turnaround time. J Digit Imaging 2008;21(1):50-8.

18. Morgan MB, Branstetter BFT, Mates J, Chang PJ, Flying blind: using a digital dashboard to navigate a complex PACS environment. J Digit Imaging 2006;19(1):69-75.

19. Korb W, Bohn S, Burgert O, Dietz A, Jacobs S, Falk V, et al. Surgical PACS for the digital operating room. Systems engineering and specification of user requirements. Stud Health Technol Inform 2006; 119:267-72.

20. Lemke HU, Osteaux M. PACS and digital imagingnew directions. Eur J Radiol 1993;17(1): 1-2.

21. Muller H, Michoux N, Bandon D, A. Geissbuhler A. A review of content-based image retrieval systems in medical applications-clinical benefits and future directions. Int J Med Inform 2004;73(1):1-23.

22. Muller H, Rosset A, Garcia A, Vallee JP, Geissbuhler A. Informatics in radiology (infoRAD): benefits of content-based visual data access in radiology. Radiographics 2005;25(3):849-58.

23. Deserno TM, Antani S, Long R. Ontology of Gaps in Content-Based Image Retrieval. J Digit Imaging 2008;22(2):202-15

24. Kahn CE Jr, Channin DS, Rubin DL. An ontology for PACS integration. J Digit Imaging 2006;19(4): 316-27.

25. Bug WJ, Astahkov V, Boline J, Fennema-Notestine C, Grethe J, GuptaA, etal. Data Federation in the biomedical informatics research network: tools for semantic annotation and query of distributed multiscale brain data. AMIA Annu Symp Proc 2008: 1220.
26. Rosset A, Spadola L, Ratib O. OsiriX: an open-source software for navigating in multidimensional DICOM images. J Digit Imaging 2004;17(3):205-16.

27. Rosset C, Rosset A, Ratib O. General consumer communication tools for improved image management and communication in medicine. J Digit Imaging 2005;18(4):270-9.

\section{Correspondence to:}

Osman Ratib MD, PhD, FAHA

Professor and Chair of Radiology

Dept. of Medical Imaging and Information Sciences

Head of Nuclear Medicine Division

University Hospital of Geneva

24, rue Micheli-du-Crest

1205 Geneva, SWITZERLAND

Tel: +41223727145

Fax: + 41223727184

E-mail: osman.ratib@hcuge.ch

\section{Historic Landmark Paper Selected by Osman Ratib for the 2009 IMIA Yearbook of Medical Informatics*}

The reference paper selected is a transcript of the first workshop held on Picture Archiving and Communication Systems (PACS) in 1982. Although the proceedings of that ' 82 conference are not available online and are hard to find, this paper is a re-edition of the discussions held at the workshop and transcribed by Prof. André Duerinckx, one of the organizer of that symposium. This milestone in the history of development of PACS clearly sets up the concepts and vision of image management in healthcare, some of which are still relevant today. Most participants mentioned in this paper were the early pioneers who contributed significantly in the development of this field. It is also at this workshop that the acronym PACS was first cornered and used ever since.

\section{Duerinckx AJ. Introduction and the PACS '82 panel discussions: Panel 1 - Equip- ment manufacturers' view on PACS and Panel 2 - The medical community's view on PACS. 1982. J Digit Imaging 2003; 16(1):32-68; discussion 29-31.}

\footnotetext{
* The complete paper can be accessed in the Yearbook's full electronic version, provided that permission has been granted by the copyright holder(s)
} 